\title{
Dinâmica populacional de quatro espécies de grandes bagres na bacia do rio Cuiabá, Pantanal norte, Brasil (Siluriformes, Pimelodidae)
}

\author{
Lúcia A. F. Mateus \& Jerry M. F. Penha
}

Laboratório de Ecologia e Manejo de Recursos Pesqueiros, Departamento de Botânica e Ecologia, Instituto de Biociências, Universidade Federal de Mato Grosso. 78060-900 Cuiabá, Mato Grosso, Brasil. E-mail: Imateus@cpd.ufmt.br

\begin{abstract}
Population dynamic of four large catfishes from Cuiabá River basin, north Pantanal, Brazil (Siluriforme, Pimelodidae). The length structure of the stocks of four species of large Pimelodidae catfishes was used to study the dynamics of their populations in the Cuiabá river basin, north Pantanal. The estimative of the growth parameters, recruitment and mortality rates for the species were obtained. Among the species studied the Barbado presented the fastest growth than the others. The Pintado, Cachara and Jaú exhibited similar growth rate. For the Barbado and the Pintado we observed two annual picks of recruitment, however the Cachara and the Jaú presented a single annual pick. The analysis of the mortality indicates that the fishing is a source of mortality more important for the Barbado than for the other species.
\end{abstract}

KEY WORDS. Fish conservation; fishery management; growth; mortality; recruitment.

RESUMO. A estrutura em comprimento dos estoques de quatro espécies de grandes bagres pimelodídeos foi utilizada para se estudar a dinâmica de suas populações na bacia do rio Cuiabá, Pantanal norte. Foram estimados os parâmetros de crescimento, recrutamento e mortalidade para as espécies. Das espécies estudadas o Barbado apresentou o crescimento mais rápido que as demais, que exibiram taxas de crescimento similares. Para o Barbado e o Pintado foram encontrados dois picos anuais de recrutamento, já o Cachara e o Jaú apresentaram um único pico anual. A análise da mortalidade indica que a pesca é uma fonte de mortalidade muito mais importante para o Barbado que para as demais espécies.

PALAVRAS-CHAVE. Conservação de peixes; crescimento; gerenciamento da pesca; mortalidade; recrutamento.

Os bagres pimelodídeos são importantes componentes nas capturas de peixes de água doce da América do Sul (NovoA 1982, Bayley \& Petrere Jr 1989, Petrere et al. 2002, Mateus et al. 2004). A família engl oba cerca de 30 gêneros e 90 espécies com ampla variação de tamanhos - desde menores que 20 até maiores que $200 \mathrm{~cm}$ (LUNDBerg \& LiTtMANn 2003).

Das sete espécies de grandes bagres presentes no Pantanal (comprimentos maiores que $50 \mathrm{~cm}$ ) quatro espécies, pertencentes a três gêneros, representaram 64\% da captura total desembarcada no mercado "Antônio Moysés Nadaf", na cidade de Cuiabá, nos anos de 2000 e 2001 (Mateus et al. 2004) e $45 \%$ das capturas realizadas em 2002 no Pantanal sul (Albuquerque et al. 2003). São elas: Pseudoplatystoma corruscans (Spix \& Agassiz, 1829), P. fasciatum (Linnaeus, 1766), Pinirampus pirinampu (Spix \& Agassiz, 1829) eZungaro jahu (Ihering, 1898).

O gênero Pseudoplatystoma Bleeker, 1862 compreende três espécies de grandes predadores distribuídos pela América do Sul tropical e subtropical. P. corruscans, o Pintado, ocorre mais ao sul, sendo presente nas bacias dos rios Paraná e São Francisco (Lundberg \& LitTMAnn 2003). Pseudoplatystoma fasciatum, o Cachara, é a espécie mais bem distribuída, sendo presente nas bacias dos rios Amazonas, Corantijn, Essequibo, Orinoco e Paraná (Lundberg \& LitTmann 2003). Pseudoplatystoma tigrinum (Valenciennes, 1840), o Surubim-Tigre, ocorre mais ao norte, sendo presente nas bacias dos rios Amazonas e Orinoco (LundBerg \& LitTMANn 2003).

O gênero Pinirampus Bleeker, 1858 é monoespecífico. Pinirampus pirinampus, o Barbado, está distribuído pelas bacias do Amazonas, Essequibo, Orinoco e Paraná (Lundberg \& LiTTMANN 2003). O gênero Zungaro Bleeker, 1858 tem duas espécies distribuídas pelos rios da América do Sul. Zungaro jahu Ihering (1898) ocorre mais ao sul, na bacia do Paraná-Paraguai. Zungaro zungaro (Humboldt, 1821) ocorre mais ao norte, na bacia dos rios Amazonas e Orinoco (Lundberg \& LitTmann 2003).

Apesar da importância dessas quatro espécies para a pesca e a dinâmica dos ecossistemas de água doce, a dinâmica de suas populações ainda é pouco entendida. No Pantanal o crescimento e reprodução do Pintado e do Cachara foram estudados por Resende et al. (1996), no Rio Miranda, Pantanal sul. Mateus \& Petrere (2004) analisaram o crescimento, mortalidade e rendimento por recruta do Pintado no Rio Cuiabá. PEIXER et al. (2006) estimaram o comprimento médio na primeira 
maturação do Barbado do Rio Paraguai, Pantanal Sul e Sı LvA (1986) estimou o comprimento na primeira maturação para Jaú do Rio Paraguai, Pantanal sul.

Nesta pesquisa a estrutura em tamanho obtida a partir dos exemplares capturados pela pesca artesanal de caráter comercial, na bacia do rio Cuiabá, Pantanal norte, para os bagres pimelodídeos Barbado, Cachara, Jaú e Pintado foi utilizada para se estudar a dinâmica populacional da parcela da população sujeita a pesca. Em particular foram obtidas estimativas dos parâmetros de crescimento individual, recrutamento e mortalidade para cada uma das quatro espécies estudadas. O objetivo deste estudo foi o de avaliar o estado de conservação e discutir alternativas para o manejo dos estoques das espécies nessa bacia hidrográfica.

\section{MATERIAL E MÉTODOS}

\section{Coleta de dados}

Medidas de comprimento furcal $(\mathrm{Lf}, \mathrm{Cm})$ foram obtidas a partir do pescado desembarcado no Mercado "Antônio Moysés Nadaf", na cidade de Cuiabá, Mato Grosso. Os dados foram coletados mensal mente entre maio e outubro de 2000 e março e abril de 2001 (de novembro/2000 a fevereiro/2001 a pesca esteve proibida em função do período de proteção a desova dos peixes). Todos os peixes foram capturados pela pesca artesanal praticada com linha e anzol na bacia do rio Cuiabá, um dos principais tributários da bacia do Alto rio Paraguai, Pantanal Mato-grossense. Para maiores detal hes sobre a área de estudos, bem como sobre as características das pescarias praticadas na região ver Penha \& MAteus (2007).

O peso total (W, gramas) de uma sub-amostra de cada espécie, também foram obtidos para o estabelecimento das relações entre o peso e o comprimento furcal. O número de exemplares pesados variou, pois foram amostrados apenas aqueles que eram desembarcados ainda não eviscerados.

Os dados de comprimento foram agrupados em classes de Lf e sua freqüência mensal tabulada. Para o Barbado o agrupamento foi em classes de $1 \mathrm{~cm}$. Para as demais espécies o Lf foi agrupado em classes de $2 \mathrm{~cm}$.

\section{Crescimento}

A relação entre o peso total e o comprimento furcal foi obtida pela expressão: $W=a \cdot L f$ b linear.

Os parâmetros $a$ e b foram estimados por regressão não

O modelo de von Bertalanffy (BerTalanfFy 1938) Fol utilizado para o ajuste da curva de crescimento para os dados de comprimento: $\mathrm{L}(\mathrm{t})=\mathrm{L}_{\infty}\left[1-\exp \left\{-\mathrm{k}\left(\mathrm{t}-\mathrm{t}_{0}\right)\right\}\right]$; onde $\mathrm{Lt}$ é o comprimento na idade $t ; L_{\infty}$ é o comprimento assintótico; $k$ é o coeficiente de crescimento e to é a idade teórica em que o comprimento ézero.

O comprimento assintótico $\left(\mathrm{L}_{\infty}, \mathrm{cm}\right)$ e o coeficiente de crescimento $\left(\mathrm{k}, \mathrm{ano}^{-1}\right)$ foram estimados pelo método SLCA (Shepherd's Length Composition Analysis; SHEPHERD 1987) incluído no FISAT software (FAO-ICLARM Stock Assessment Tools;
Gayanilo et al. 1994). A estimativa de t0 (anos) foi obtida pela relação empírica: $\log _{10}\left(-t_{0}\right)=-0,392-0,275 \log _{10} L_{\infty}-1,038 \log _{10} k$ (PAuly 1979). O índice de performance de crescimento $\left(\varnothing^{\prime}\right)$ foi estimado conforme a expressão: $\varnothing^{\prime}=\log _{10}(k)+2 \log _{10}\left(L_{\infty}\right)$ (Pauly \& MunRo 1984)

O comprimento médio na primeira maturação $(\mathrm{Lm}, \mathrm{cm})$ foi estimado a partir do L $\infty$ pela equação empírica: $\log _{10} \mathrm{Lm}=0,898 * \log _{10}\left(\mathrm{~L}_{\infty}\right)-0,0781$ (FROESE \& BinOHLAN 2000).

A idade média de primeira maturação (tm), então pode ser estimada a partir de Lm, usando-se a equação de von Bertalanffy, rearranjada para $\left.t: t_{m}=-1 / k\left\{\ln \left(L_{\infty}-L m / L_{\infty}\right)\right]-k t o\right\}$.

O comprimento ótimo (Lopt, $\mathrm{cm}$ ) é definido como a classe decomprimento ondeo produto do número de indivíduos vezes o seu peso médio alcança o valor máximo. Este pode ser estimado a partir de $L_{\infty}$ pela equação empírica: $\log _{10} \mathrm{~L}_{\text {opt }}=1,0003 * \log _{10}\left(\mathrm{~L}_{\infty}\right)-0,2161$ (FroESE \& BinOHLAN 2000).

\section{Recrutamento para a pesca}

A distribuição de freqüência de classes de comprimento e os parâmetros de crescimento estimados para cada espécie foram utilizados para identificar o número de pulsos de recrutamento por ano e avaliar a relativa importância destes quando comparados entre si, através da rotina incluída no FISAT (Gayanilo \& Pauly 1997).

\section{Mortalidade}

A taxa instantânea de mortalidade natural (M) foi estimada pela relação empírica entre $L_{\infty}, \mathrm{k}$ e a temperatura média anual $\left({ }^{\circ} \mathrm{C}\right)$ do hábitat. Onde T, para peixes de água doce, baseia-se na suposição de que a temperatura média anual superficial da água corresponde à temperatura média anual do ar na mesma área (PAULY 1980). Segundo dados do 9o Distrito de Metereologia a temperatura média anual para a região no período foi de 26 으. O modelo empírico é: $\ln (M)=-0,0152-0,279 \ln \left(L_{\infty}\right)+0,6543 \ln (k)+0,463 \ln (T)$.

Para diminuir as incertezas associadas com as estimativas, a taxa instantânea de mortalidade total (Z) foi obtida por meio de três modelos:

1) Beverton \& Holt (1957): $Z=k\left(L_{\infty}-L\right) /(L-L C)$, onde (LC é o comprimento médio de primeira captura, quando os peixes então são recrutados para a pesca éL é o comprimento médio a partir de Lc (KING 1995). Sendo a variância de $Z$ estimada por: $\operatorname{Var}(Z)=k^{2}\left[(Z / k)(Z / k+1)^{2} / n .(Z / k+2)\right]$ (Wetherall et al. 1987); onde n é o tamanho da amostra.

2) Ault \& EHRHARDT (1991) - uma correção apresentada para o método de Beverton $\&$ Holt, onde $L_{\infty}$ é substituído pelo maior comprimento na amostra $\left(L_{\max }\right)$, o que leva à seguinte expressão:

$\left.\left[\left(\mathrm{L}_{\infty}-\mathrm{L}_{\max }\right) /\left(\mathrm{L}_{\infty}-\mathrm{LC}\right)\right]^{\mathrm{Z} / \mathrm{k}}=\left\{\mathrm{Z}(\mathrm{LC}-\mathrm{L})+\mathrm{k}\left(\mathrm{L}_{\infty}-\mathrm{L}\right)\right] /\left[\mathrm{Z}\left(\mathrm{L}_{\max }-\mathrm{L}\right)+\mathrm{k}\left(\mathrm{L}_{\infty}-\mathrm{L}\right)\right]\right\}$

3) SSENTONGO \& LARKIN (1973):

$Z=[(n . k) /(n+1)] .\left\{\ln \left[L_{\infty}-L c /\left(L_{\infty}-L\right)\right]\right\}^{-1} ;$ onde $n$ é o tamanho da amostra; (Lc é o comprimento médio de primeira captura e ( $L$ é o comprimento médio obtido na distribuição de freqüência. Com a variância de $Z$ estimada como segue: 
$\left.\operatorname{Var}(Z)=k^{2}\left\{n /(n+1)^{2}\right]\right\}\left\{\left\{n\left[\ln \left(\left(L_{\infty}-\mathrm{LC}\right) /\left(\mathrm{L}_{\infty}-\mathrm{L}\right)\right)\right]\right\}^{2}\right.$.

A taxa instantânea de mortalidade por pesca $(F)$ foi estimada como a diferença entre a taxa instantânea de mortalidade total (Z) e a taxa instantânea de mortalidade natural (M).

A taxa finita de sobrevivência $\left(\mathrm{S}, \mathrm{ano}^{-1}\right)$ pode ser estimada por: $S=e^{z}$ ou $S(\%)=100 \cdot e^{z}$

\section{RESULTADOS}

\section{Estrutura em tamanho}

Foram obtidos medidas de 908 Barbados, 1283 Cacharas, 488 Jaús e 1445 Pintados durante o período de amostragem. O comprimento dos Barbados variou entre 43 e $87 \mathrm{~cm}\left(\mathrm{~L}_{\text {mediana }}=\right.$ 64,0; $\left.L_{\text {médio }}=63,6 ; S D=5,4\right)$, o dos Cacharas entre 60 e $114 \mathrm{~cm}$ $\left(L_{\text {mediana }}=83,0 ; L_{\text {médio }}=84,2 ; S D=6,35\right)$, o dos Jaús entre 84 e 163 $\mathrm{cm}\left(\mathrm{L}_{\text {mediana }}=106 ; \mathrm{L}_{\text {médio }}=107,5 ; \mathrm{SD}=14,55\right)$ e dos Pintados entre 57 e $140 \mathrm{~cm}\left(L_{\text {mediana }}=95 ; L_{\text {médio }}=97,7 ; S D=12,87\right)$ (Fig. 1). O peso dos Barbados da amostra variou entre 800 e $4800 \mathrm{~g}\left(\mathrm{~W}_{\text {médio }}=2100\right.$; $\mathrm{SD}=760 ; \mathrm{n}=68)$, o dos Cacharas entre 1000 e $15300 \mathrm{~g}\left(\mathrm{~W}_{\text {médio }}=\right.$ 5900; SD =1980; $\mathrm{n}=305)$, o dos Jaús entre 8000 e $42000 \mathrm{~g}$ (W médio $_{\text {. }}$ =21400; SD =9950; $\mathrm{n}=31$ ) e o dos Pintados entre 1300 e 41000 $\mathrm{g}\left(\mathrm{W}_{\text {médio }}=10900 ; \mathrm{SD}=7240 ; \mathrm{n}=992\right)$ (Fig. 1).

\section{Crescimento}

As estimativas do parâmetro b da relação entre o peso e o comprimento indicam que três das quatro espécies estudas apresentaram crescimento isométrico (os interval os de confiança das estimativas incluem o valor 3). Para o Barbado a relação entre o peso e o comprimento pode ser descrita pela seguinte equação: $\mathrm{W}=0,014 * \mathrm{~L}^{2,945}$ (Intervalo de confiança do $\mathrm{b}=2,724-3,166 ; \mathrm{R}^{2}=$ $0,915 ; n=68)$. Para o Cachara a relação entre o peso e o comprimento pode ser descrita pela seguinte equação: $W=0,006 * L^{3,126}$ (Intervalo de confiança do $b=2,989-3,262 ; R^{2}=0,872 ; n=305$ ). E para o Jaú essa relação pode ser descrita pela equação: $W=$ $0,005 * L^{3,228}$ (Intervalo deconfiança do $b=2,931-3,526 ; R^{2}=0,952$; $\mathrm{n}=31$ ). Apenas o Pintado apresentou valor estimado do $\mathrm{b}>3$, indicando crescimento alométrico positivo ( $\mathrm{W}=0,005^{*} \mathrm{~L}^{3,172}$; Intervalo de confiança do $b=3,119-3,225 ; R^{2}=0,949 ; n=990$ ).

Os parâmetros de crescimento, L $\infty$ e k obtidas pelo método SLCA foram estimados, respectivamente em $90,6 \mathrm{~cm}$ e 0,30 ano-1 para o Barbado; 122,4 cm e 0,130 ano-1 para o Cachara; $172 \mathrm{~cm}$ e 0,128 ano-1 para o Jaú e 149,7 cm e 0,127 ano-1 para o Pintado. Para as quatro espécies o score obtido na estimativa dos parâmetros foi igual a 1,0, indicando uma satisfatória combinação de estimativas a partir da análise de distribuição de freqüência. $O t_{0}$ foi estimado em $-0,41$ ano para o Barbado, $-0,898$ para o Cachara, -0,832 para o Jaú e -0,871 para o Pintado. O cálculo do índice de performance de crescimento $\left(\varnothing^{\prime}\right)$ variou de 3,28 a 3,57, sendo a média de 3,430 (Intervalo de Confiança = 3,239-3,621) e o Coeficiente de Variação de 3,5\%. O valor indica uma satisfatória estimativa dos parâmetros de crescimento, uma vez que para uma mesma família este índice não pode variar muito mais que $5 \%$ (Tab. I).

Os ajustes do modelo de crescimento de von Bertalanffy, indicam que o menor comprimento assintótico $(\mathrm{L} \infty)$ é alcança- do pelo Barbado e o maior pelo Jaú, respectivamente. O Cachara e o Pintado apresentaram comprimentos assintóticos intermediários e o Barbado apresentou o crescimento mais acelerado em comparação com as outras espécies aqui estudadas. Os vaIores de k para o Cachara, o Jaú e o Pintado são numericamente muito próximos, indicando que essas diferentes espécies se aproximam de seus respectivos comprimentos assintóticos com a mesma velocidade.

As estimativas do comprimento médio de primeira maturação ( $\mathrm{Lm}$ ) indicam que o Barbado tem sua primeira maturação em torno dos dois anos, quando atinge um comprimento médio de 47,8 cm (Tab. I). O Cachara, o Jaú e o Pintado maturam, aproximadamente, na mesma idade, em torno dos 4,6 anos, porém com comprimentos diferenciados. A quantidade de indivíduos capturados pela pesca comercial que se encontram abaixo do Lm estimado é muito pequena para as quatro espécies aqui estudadas, representando 0,33\% da captura do Barbado, 0,08\% do Cachara e 0,34\% do Pintado. Para o Jaú todos os indivíduos capturados estavam acima do Lm estimado (Tab. I e Fig. 2).

Em relação ao $L_{\text {opt }}$ constata-se que a maioria dos indivíduos dos estoques do Barbado e do Cachara estão sendo capturados acima deste valor, não havendo sobreposição do intervalo de confiança do $L_{\text {opt }}$ com o intervalo de confiança do comprimento médio amostrado para estas espécies. Quanto ao Jaú e o Pintado, considerando o intervalo de confiança, o comprimento médio não difere significativamente do comprimento ótimo (Tab. I e Fig. 2).

\section{Recrutamento para a pesca}

A análise das curvas de recrutamento revelou picos bem definidos para as quatro espécies, associados principalmentecom os períodos hidrológicos de enchente e vazante do rio Cuiabá. Para o Barbado e o Pintado foram encontrados dois picos anuais, já o Cachara e o Jaú apresentaram um único pico anual (Fig. 3). A intensidade do recrutamento para o Barbado foi maior no mês de julho (período de vazante e seca), apresentando um pico mais suave nos meses de fevereiro e março (período de cheia). Para o Pintado a intensidade do recrutamento foi mais forte em outubro (período de transição entre a seca e a enchente), havendo um outro pico mais suave no mês de maio (período de vazante). O Cachara apresentou maior intensidade de recrutamento entre os meses de março, abril e maio (transição entre cheia e vazante). O Jaú também apresentou um único pico, que ocorreu nos meses de novembro, dezembro e janeiro (enchente).

\section{Mortalidade}

O Cachara, o Pintado e o Jaú apresentaram estimativas da taxa instantânea de mortalidade natural muito próximos entre si, em torno de 0,3 ano-1, enquanto que o Barbado apresentou taxa instantânea de mortalidade duas vezes mais alta, próxima a 0,6 ano-1. O Barbado também apresentou a maior taxa instantânea de mortalidade total - $Z$ (média dos três modelos $=1,155$ ano-1 $^{-1}$, seguido pelo Cachara $\left(0,657\right.$ ano-1 $\left.^{-1}\right)$, pelo Jaú $\left(0,465\right.$ ano $\left.^{-1}\right)$ e pelo Pintado $\left(0,427 \mathrm{ano}^{-1}\right)$ (Tab. II). Os mo-

Revista Brasileira de Zoologia 24 (1): 87-98, março 2007 

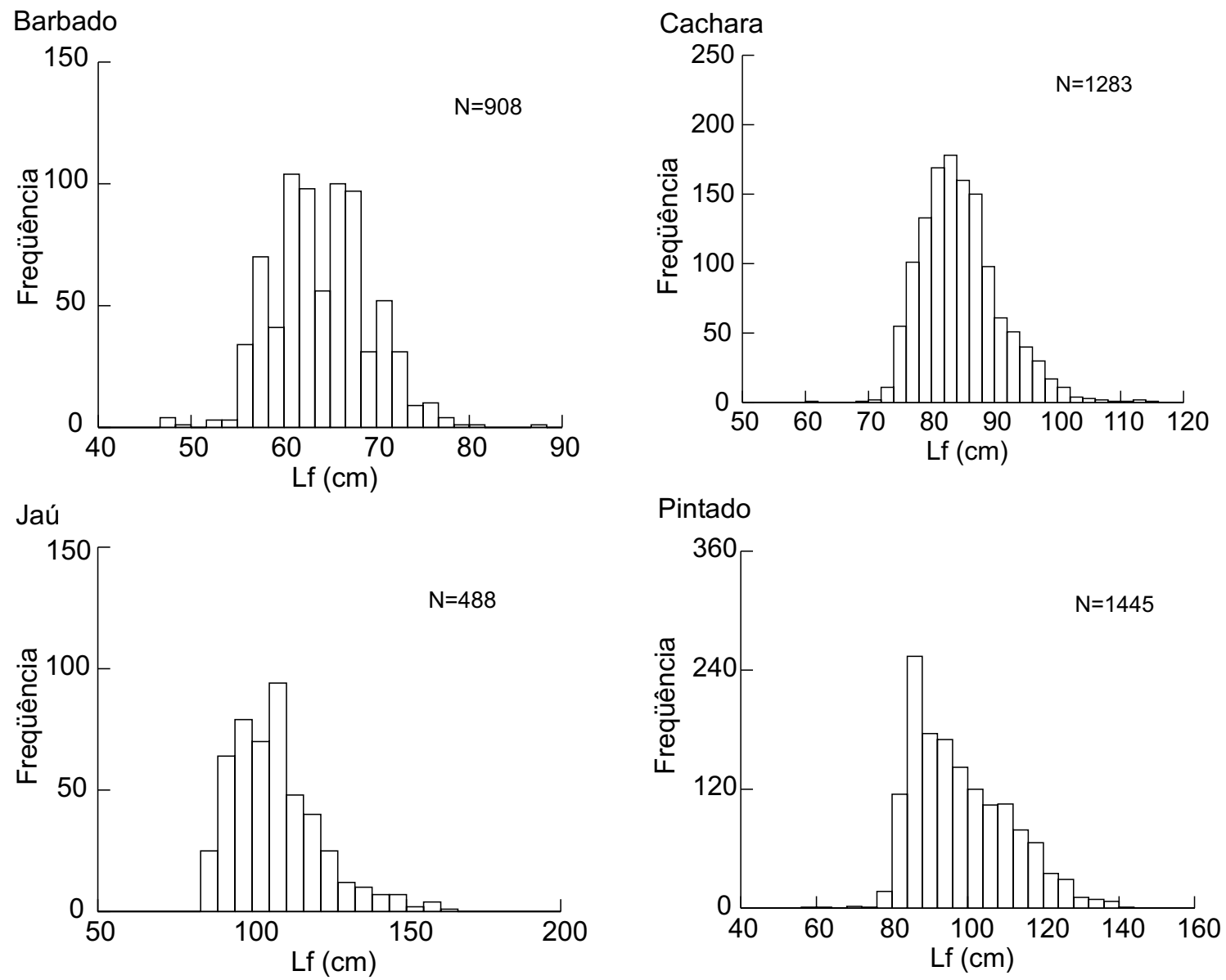

Figura 1. Distribuição de freqüências de Lf (cm) para os estoques dos quatro grandes bagres na bacia do rio Cuiabá.

Tabela I. Estimativas dos parâmetros populacionais para os estoques dos quatro grandes bagres na bacia do rio Cuiabá. Idade da primeira maturação $(\mathrm{Im})$; comprimento médio da primeira maturação $(\mathrm{Lm})$; comprimento ótimo $\left(\mathrm{L}_{\mathrm{opt}}\right)$ Entre parênteses estão os intervalos de confiança a $95 \%$ de $L m$ e $L_{\text {ont }}$.

\begin{tabular}{lcccc}
\hline \multicolumn{1}{c}{ Parâmetros } & Barbado & Cachara & Jaú & Pintado \\
\hline Lo (cm) & 90,6 & 122,4 & 172,0 & 149,7 \\
k (ano-1) & 0,30 & 0,130 & 0,128 & 0,127 \\
t0 (ano) & $-0,410$ & $-0,898$ & $-0,832$ & $-0,871$ \\
$\varnothing^{\prime}$ & 3,39 & 3,29 & 3,58 & 3,45 \\
Lc (cm) & 58,5 & 78,0 & 94,0 & 84,0 \\
L (cm) & 65,3 & 85,5 & 111,2 & 99,5 \\
Lmax (cm) & 87,0 & 114,0 & 163,0 & 140,0 \\
Lopt (cm) & $55,2(50,1-60,7)$ & $74,5(67,5-82,3)$ & $104,7(94,4-116,2)$ & $91,2(82,3-100,9)$ \\
Lm (cm) & $47,8(43,7-52,3)$ & $62,6(56,3-69,6)$ & $85,0(74,8-96,5)$ & $75,0(66,7-84,5)$ \\
Im (anos) & 2,09 & 4,61 & 4,49 & 4,60 \\
\hline
\end{tabular}

delos de BeVERTon \& Holt e Ault \& EHRHARDt forneceram estimativas de taxa de mortalidade total (Z) muito próximas para cada uma das quatro espécies. As estimativas de $Z$ baseadas no com- primento médio foram relativamente mais altas quando o modelo de Ssentongo \& LARKIN foi utilizado. Considerando a média de Z cal culada a partir dos métodos baseados em comprimento 
Barbado

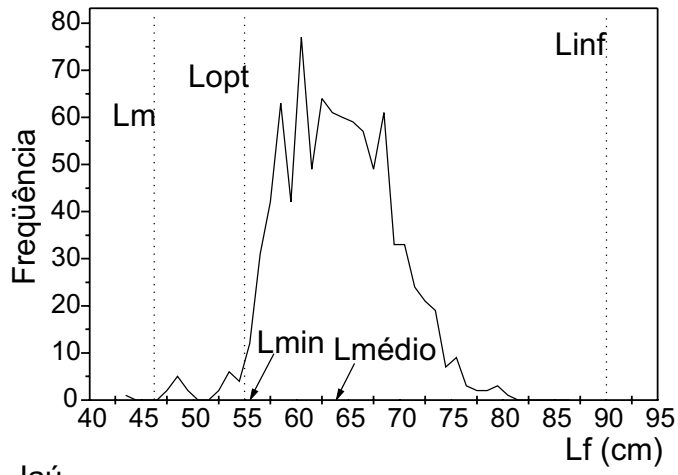

Jaú

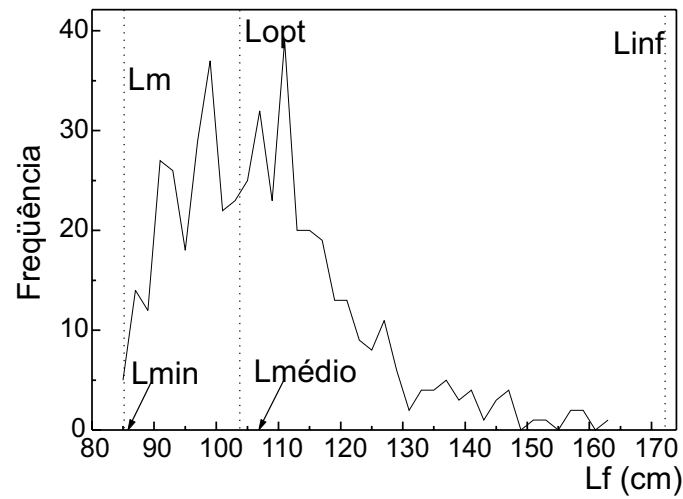

\section{Cachara}
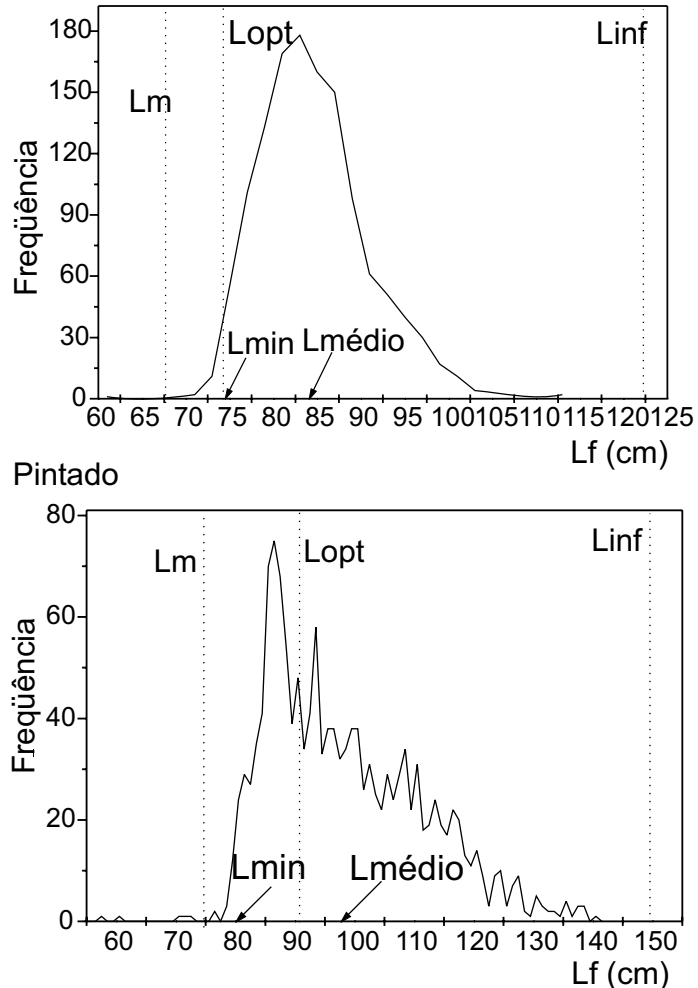

Figura 2. Distribuição de freqüência de comprimento furcal, com os comprimentos de primeira maturação (Lm, cm), comprimento ótimo $\left(\mathrm{L}_{\mathrm{opt}}, \mathrm{cm}\right)$, comprimento mínimo permitido $\left(\mathrm{L}_{\text {min' }}, \mathrm{cm}\right)$, comprimento médio (Lmédio, $\left.\mathrm{cm}\right)$ e comprimento assintótico $(\mathrm{L} \infty$, $\mathrm{cm}$ ) para os estoques dos quatro grandes bagres na bacia do rio Cuiabá.

Tabela II. Estimativas de mortalidade natural $\left(M\right.$, ano $\left.{ }^{-1}\right)$ e total $\left(Z\right.$, ano $\left.^{-1}\right)$ e estatísticas descritivas dos valores de $Z$ (ano $\left.{ }^{-1}\right)$ estimados pelos métodos baseados em comprimento médio de captura para os estoques dos quatro grandess bagres na bacia do rio Cuiabá. (IC) Intervalo de confiança, (se) erro padrão, (CV) coeficiente de variação.

\begin{tabular}{|c|c|c|c|c|c|c|}
\hline & \multirow{2}{*}{$\frac{\text { Mortalidade natural }\left(\mathrm{M} \mathrm{ano}^{-1}\right)}{\text { PAULY (1980) }}$} & \multicolumn{5}{|c|}{ Mortalidade total $\left(Z\right.$ ano $\left.{ }^{-1}\right)$} \\
\hline & & $\begin{array}{l}\text { BeVERTON \& Holt } \\
(1956) \pm \text { se }\end{array}$ & $\begin{array}{c}\text { AuLt \& EHRHARDT } \\
(1991)\end{array}$ & $\begin{array}{c}\text { SSENTONGO \& LARKIN } \\
(1973) \pm \mathrm{se}\end{array}$ & Média (IC) & CV (\%) \\
\hline Barbado & 0,576 & $1,112 \pm 0,074$ & 1,102 & $1,252 \pm 0,004$ & $1,155(0,949-1,360)$ & 7,2 \\
\hline Cachara & 0,307 & $0,641 \pm 0,036$ & 0,631 & $0,704 \pm 0,001$ & $0,657(0,565-0,750)$ & 5,7 \\
\hline Jaú & 0,276 & $0,453 \pm 0,041$ & 0,433 & $0,513 \pm 0,003$ & $0,465(0,366-0,565)$ & 8,6 \\
\hline Pintado & 0,286 & $0,411 \pm 0,022$ & 0,401 & $0,471 \pm 0,001$ & $0,427(0,335-0,520)$ & 8,7 \\
\hline
\end{tabular}

médio observa-se um coeficiente de variação relativamente baixo para cada uma das espécies (Tab. II).

A partir das estimativas de $Z$ e $M$ foi estimada a mortalidade por pesca (F) atual, considerando $M$ constante e Z estimado pelos diferentes métodos (Tab. III). Comparando-se os valores estimados para $\mathrm{M}$ e $\mathrm{F}$ é possível concluir que a pesca foi a fonte mais importante de mortalidade $(\mathrm{F}>\mathrm{M})$ para o estoque do Cachara, sendo responsável por $52,8 \%$ da mortalidade total (Fig. 4). A pesca e a mortalidade natural agem praticamente na mesma proporção no estoque do Barbado, ou seja, $F \cong M$. Por outro lado a pesca é menos importante que as outras fontes de mortalidade ( $\mathrm{F} \triangleleft \mathrm{M})$ para os estoques do Jaú e Pintado (Fig. 4). Desse modo, considerando-se a relação entre $F$ e $M$, pode-se concluir que o estoque do Cachara vem sendo submetido a maior pressão pesqueira do que os outros bagres em questão.

Uma maneira alternativa de se analisar o efeito da mortalidade é se concentrar no seu oposto, a sobrevivência. Nesse aspecto o Barbado é a espécie que tem a mais baixa taxa de sobre- 


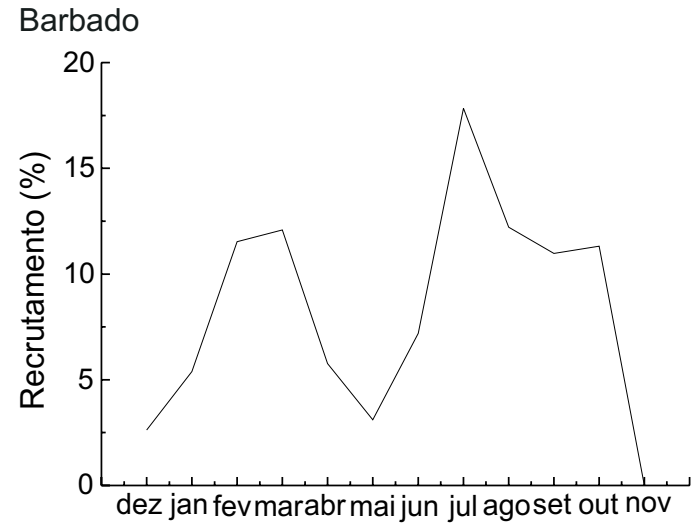

Jaú

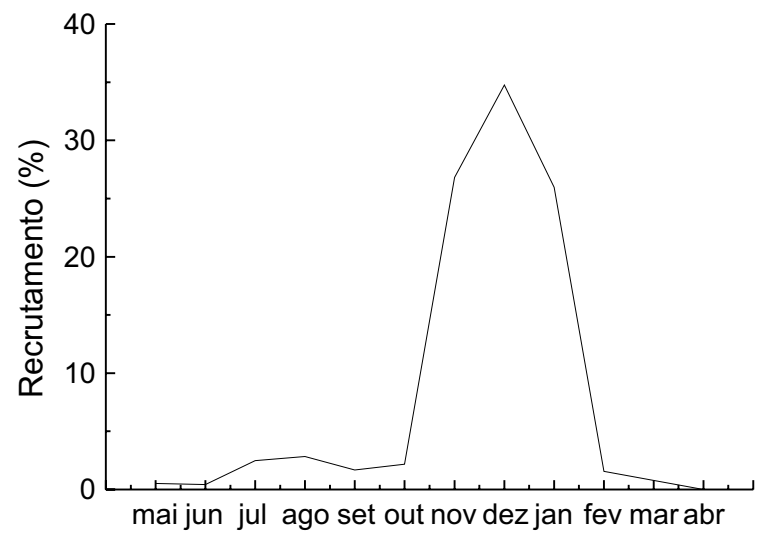

Meses

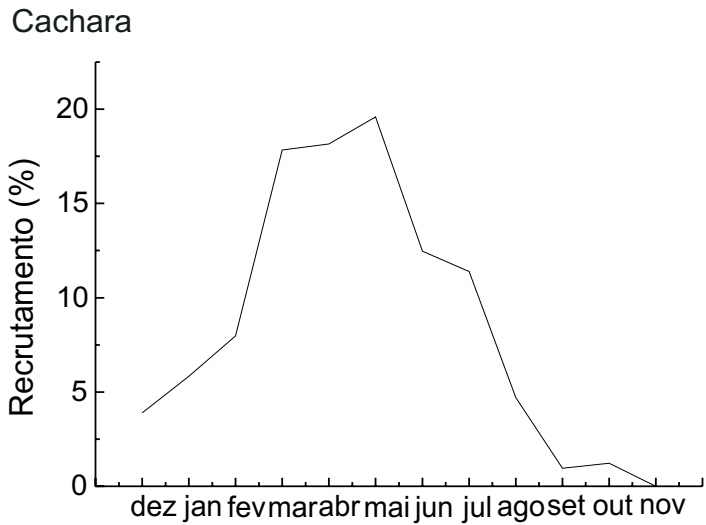

Pintado

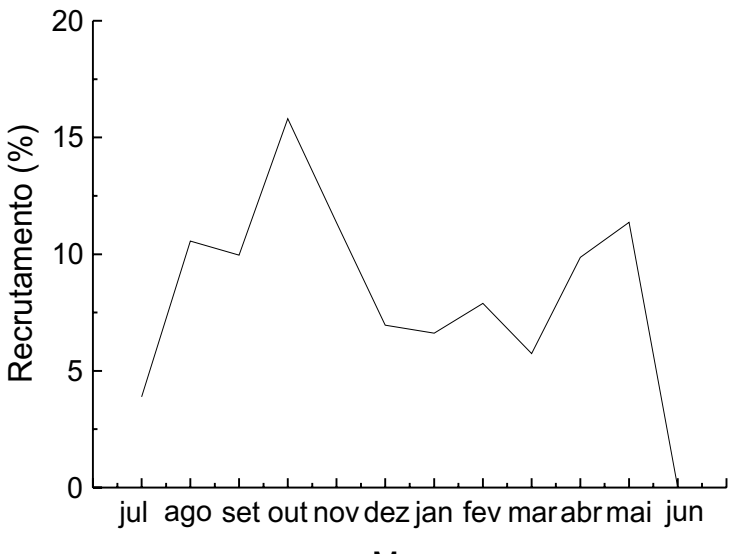

Figura 3. Intensidade do recrutamento para os estoques dos quatro grandes bagres na bacia do rio Cuiabá.

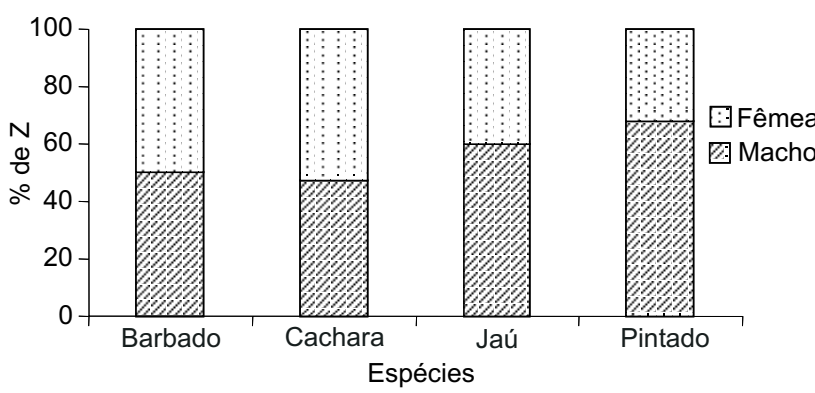

Figura 4. Porcentagem de mortes provocadas por causas naturais (M) e pela pesca (F), para os estoques do Barbado, Cachara, Jaú e Pintado, na bacia do rio Cuiabá, Mato Grosso.

vivência, seguido pelo Cachara, Jaú e Pintado (Tab. IV). A porcentagem média dos indivíduos que sobrevivem de um intervalo detempo para outro para o Barbado é de $31,5 \%$, para o Cachara de $51,8 \%$, para o Jaú de $62,8 \%$ e para o Pintado de $65,2 \%$.

Se apenas a mortalidade natural estivesse agindo nestes estoques a taxa de sobrevivência do Barbado seria de $56 \%$, a do
Tabela III. Estimativas da atual mortalidade por pesca $\mathrm{F}\left(\mathrm{ano}^{-1}\right)$, para os estoques estudados.

\begin{tabular}{lrrrr}
\hline \multicolumn{1}{c}{ Z utilizado } & Barbado & Cachara & Jaú & Pintado \\
\hline BeVERTon \& Holt (1956) & 0,532 & 0,331 & 0,173 & 0,121 \\
Ault \& EHRHARDT (1991) & 0,522 & 0,321 & 0,153 & 0,111 \\
Ssentongo \& LarKIN (1973) & 0,670 & 0,390 & 0,230 & 0,180 \\
Média & 0,575 & 0,347 & 0,185 & 0,137 \\
SD & 0,083 & 0,037 & 0,040 & 0,037 \\
CV (\%) & 14,400 & 10,700 & 12,100 & 27,200 \\
\hline
\end{tabular}

Tabela IV. Estimativas da taxa de sobrevivência (S,\%), para os estoques estudados.

\begin{tabular}{lcccc}
\hline \multicolumn{1}{c}{ Z utilizado } & Barbado & Cachara & Jaú & Pintado \\
\hline Beverton \& Holt (1956) & 32,9 & 52,7 & 63,6 & 66,3 \\
Ault \& EHRHARDt (1991) & 33,2 & 53,2 & 64,8 & 66,9 \\
Ssentongo \& LARKIn (1973) & 28,6 & 49,6 & 60,0 & 62,5 \\
Média & 31,5 & 51,8 & 62,8 & 65,2 \\
\hline
\end{tabular}


Cachara 73,3\%, do Jaú 75,6\% e a do Pintado 74,8\%. Assim, a redução na taxa de sobrevivência em decorrência da pesca é mais acentuada para o estoque do Barbado (24,5\%) e mais amena para o estoque do Pintado, a qual é menor que $10 \%$.

\section{DISCUSSÃO}

\section{Estrutura em tamanho}

Ao longo do tempo se tem notado que, usualmente, existem mais peixes pequenos nas capturas do que grandes. $\mathrm{O}$ padrão é: poucos jovens, pico para idades intermediárias e poucos velhos. As possíveis razões para isso são: (I) peixes jovens são menos vulneráveis, pois ainda não estão completamente recrutados para a pesca; (II) peixes velhos são menos abundantes na população (devido à mortalidade) e ainda se tornam menos vulneráveis ao aparel ho por várias razões (HILBORN \& W ALTERS 1992).

Neste estudo a distribuição de freqüência de tamanho das capturas para todas as espécies foi condizente com o padrão descrito anteriormente. Neste caso, somando-se ao fato dos peixes menores não estarem completamente recrutados, existe uma restrição legal à captura de indivíduos abaixo de um certo tamanho mínimo. Assim esta distribuição de comprimentos pode ser considerada representativa da porção dos estoques sujeita a pesca, porém não representa idealmente a população como um todo. No entanto, para o propósito final desta análise que é a avaliação dos estoques, pode-se considerar esta amostra satisfatória (Jones 1984, Gayanilo \& PAULY 1997).

Foi observado que a comercialização de peixes jovens no Mercado de Cuiabá é pequena. Assim deste ponto de vista podese considerar que a pressão de pesca não é, ainda, uma ameaça aos estoques. Entretanto a captura clandestina de peixes jovens ocorre e deve ser considerada. Por outro lado, a pressão sobre os peixes grandes pode vir a ser um problema, uma vez que a maioria das pescarias remove indivíduos grandes e ve Ihos do estoque primitivo, assim truncando a sua pirâmide natural de classes etárias, reduzindo sua longevidade natural e característica. Teoricamente isto pode levar a redução do fitness médio dos indivíduos do estoque a despeito do crescimento compensatório dos sobreviventes (LONGHUSRST 2002).

\section{Crescimento}

Nesta pesquisa os parâmetros de crescimento foram obtidos pela análise da distribuição de freqüência de comprimentos, considerando que a reprodução das espécies avaliadas é anual, e concentrada em um período do ano relativamente curto (Resende et al. 1995, Reina et al. 1995, Peixer et al. 2006), permitindo assim a identificação de diferentes modas nas distribuições, condição essencial para este tipo de análise (GALLuCCI et al. 1996, Gayanilo \& Pauly 1997, Sparre \& Venema 1997, Quinn ॥ $\&$ DeRISO 1999). O método aqui utilizado (SLCA) é diretamente baseado na composição de comprimentos, ou seja, sem a prévia transformação dos comprimentos em idades relativas. É um método similar em conceito ao ELEFAN (Electronic Length Frequency Analysis), mas com algorítimo mais simples e fácil de computar. Ambos encontram dificuldades no ajuste quan- do as distribuições apresentam múltiplos picos e são mais eficientes quando um dos parâmetros, usualmente $L \infty$, pode ser estimado a priori, ou aproximado, de uma estreita amplitude de valores. SLCA apresenta-se mais robusto que o ELEFAN em casos de aumento da variância no comprimento na idade (Rosenberg \& Beddington 1987) e para espécies que alcançam el evados tamanhos assintóticos (ISAAC 1990), como é o caso dos pimelodídeos aqui analisados.

Os parâmetros de crescimento obviamente diferem entreas espécies, mastambém podem variar intraespecificamente, ou seja, a mesma espécie pode ter valores diferentes em diferentes partes de sua distribuição, dependendo das condições ambientais. Assim, o Cachara, a mais bem estudada das quatro espécies, apresenta $L \infty$ e $k$ que variam, respectivamente, entre $178 \mathrm{~cm}$ e 0,33 ano-1, na região do Médio Amazonas (Ruffino \& Isaac 1995) a 90,05 cm 0,383 ano-1, no Rio Mamoré, Bolívia (Loubens \& PanflLI 2000). O Pintado apresenta L $\infty$ e k que variam, respectivamente, entre $183,2 \mathrm{~cm}$ e 0,085 ano-1, no Rio Cuiabá (Mateus \& Petrere 2004) e 105,75 cm e 0,2445 ano-1, no Rio Miranda, Pantanal sul (Resende et al. 1996). Para o Jaú o Lœ e o k variam entre $196 \mathrm{~cm}$ e 0,0995 ano-1, no Alto rio Meta, Colômbia (Reina et al. 1995) e $172 \mathrm{~cm}$ e 0,128 ano-1, neste estudo(Tab. V). Contudo essa variação precisa ser analisada com ressalvas, visto que as estimativas dos parâmetros foram obtidas por diferentes métodos, tanto a partir de estudos de anéis de crescimento, quanto análise de distribuição de freqüência de comprimento.

O exame dos estimadores dos parâmetros de crescimento da curva de von Bertalanffy de várias espécies sugere que há uma relação inversa entre $k$ e $L \infty$, ou seja, peixes com alto valor de $L \propto$ tendem a ter baixo valor de k e vice-versa (JoBLING 1995, Sparre \& Venema 1997). Para o Cachara, o Jaú e o Pintado o que se encontrou foi um $k$ aproximadamente igual, porém com Lœ numericamente diferente para cada espécie. Já para o Barbado o L $\infty$ foi relativamente menor e o $\mathrm{k}$ relativamente maior em comparação com as outras espécies. O parâmetro $k$ é um parâmetro de curvatura que determina a velocidade com que o peixe se aproxima do $L \infty$ e pode ser considerado como uma função da taxa de crescimento, e está relacionado com a taxa metabólica dos peixes (Pauly 1979). Assim, entre as espécies estudadas é possível concluir que o Cachara, o Jaú e o Pintado são espécies que possuem uma baixa velocidade de crescimento, atingem grandes comprimentos e conseqüentemente al cançam alta longevidade, enquanto que o Barbado é uma espécie de crescimento relativamente mais rápido. Ainda, o Cachara, o Jaú e o Pintado, que apresentam aproximadamente o mesmo k, crescem com velocidade semelhante, ou seja, atingem seu respectivo comprimento assintótico na mesma idade.

\section{Tamanho na primeira maturação e tamanho ótimo}

O inicio da maturação sexual representa um período crítico para os animais que continuam a crescer após a maturidade, pois a partir daí passa a existir um potencial conflito entre a alocação de recursos entre a sobrevivência, crescimento e re

Revista Brasileira de Zoologia 24 (1): 87-98, março 2007 
Tabela V. Comparação dos parâmetros de crescimentos encontrados na literatura com aqueles estimados por este estudo para os quatro grandess bagres da bacia do rio Cuiabá, Mato Grosso.

\begin{tabular}{|c|c|c|c|c|c|c|c|c|c|}
\hline Espécie & L & $\operatorname{Lmax}(\mathrm{cm})$ & $\mathrm{L} \infty(\mathrm{cm})$ & $\mathrm{K}\left(\mathrm{ano}^{-1}\right)$ & $\varnothing^{\prime}$ & $\mathrm{N}$ & Método & Local & Fonte \\
\hline P. pirinampu & Lf & 87 & 90,6 & 0,30 & 3,3914 & 908 & $\begin{array}{l}\text { Distribuiç̧ão de } \\
\text { freqüência }\end{array}$ & $\begin{array}{l}\text { Rio Cuiabá } \\
\text { Pantanal/MT }\end{array}$ & Este estudo \\
\hline \multirow[t]{7}{*}{ P. fasciatum } & Lt & 120 & $\begin{array}{l}178 \\
169\end{array}$ & $\begin{array}{l}0,33 \\
0,27\end{array}$ & $\begin{array}{r}4,0194 \\
3,8871\end{array}$ & \multirow[t]{2}{*}{698} & Distrib de freqüência & Amazonas Tapajós & $\begin{array}{l}\text { RuFFINO \& ISAAC } \\
\text { (1995) (2 anos) }\end{array}$ \\
\hline & Lt & & 119 & 0.15 & 3,3272 & & $\begin{array}{l}\text { Distribuição de } \\
\text { freqüência }\end{array}$ & Rio Mamoré & PAYNe (1987) \\
\hline & Ls & $\begin{array}{c}100 \\
0^{x} 83\end{array}$ & $\begin{array}{l}130 \\
103\end{array}$ & $\begin{array}{l}0,098 \\
0,122\end{array}$ & $\begin{array}{l}3,2182 \\
3,1135\end{array}$ & 44 & Anéis em vértebras & $\begin{array}{l}\text { Rio Apure } \\
\text { Venezuela }\end{array}$ & Red (1983) \\
\hline & Ls & $\begin{array}{l}+95 \\
\sigma^{x} 80\end{array}$ & $\begin{array}{l}103,1 \\
77,0\end{array}$ & $\begin{array}{l}0,327 \\
0,439\end{array}$ & $\begin{array}{l}3,4004 \\
3,4154\end{array}$ & 128 & Anéis em vértebras & $\begin{array}{l}\text { Rio Mamoré } \\
\text { Amazônia Boliviana }\end{array}$ & $\begin{array}{l}\text { LOUBEnS \& PANFIU } \\
(2000)\end{array}$ \\
\hline & Ls & 90 & 우 94,5 & 0,271 & 3,3851 & 56 & Anéis em vértebras & $\begin{array}{l}\text { Rio Miranda } \\
\text { Pantanal/MS }\end{array}$ & RESENDE et al. (1996) \\
\hline & Lf & 99 & 120 & 0,165 & 3,3758 & 106 & Anéis em espinhos & $\begin{array}{l}\text { Rio Cuiabá } \\
\text { Pantanal/MT }\end{array}$ & $\begin{array}{l}\text { ESTUPIÑÁN \& MATEUS } \\
\text { (em preparação) }\end{array}$ \\
\hline & Lf & 114 & 122,4 & 0,131 & 3,2895 & 1283 & $\begin{array}{l}\text { Distribuição de } \\
\text { freqüência }\end{array}$ & $\begin{array}{l}\text { Rio Cuiabá } \\
\text { Pantanal/MT }\end{array}$ & Este estudo \\
\hline \multirow[t]{2}{*}{ P. luetkeni } & Ls & $\begin{array}{l}\text { + } 165 \\
\sigma^{\top} 138\end{array}$ & $\begin{array}{l}218 \\
174\end{array}$ & $\begin{array}{c}0,09 \\
0,109\end{array}$ & $\begin{array}{l}3,6312 \\
3,5185\end{array}$ & 107 & Anéis em espinhos & $\begin{array}{l}\text { Alto rio Meta } \\
\text { Colômbia }\end{array}$ & Rena et al. 1995 \\
\hline & Lf & 163 & 172 & 0,128 & 3,5783 & 488 & $\begin{array}{l}\text { Distribuição de } \\
\text { freqüência }\end{array}$ & $\begin{array}{l}\text { Rio Cuiabá } \\
\text { Pantanal/MT }\end{array}$ & Este estudo \\
\hline \multirow[t]{3}{*}{ P. corruscans } & LS & 125 & $\begin{array}{l}\text { of } 111,5 \\
\sigma^{x} 100,0\end{array}$ & $\begin{array}{l}0,274 \\
0,215\end{array}$ & $\begin{array}{l}3,5323 \\
3,3324\end{array}$ & 248 & Anéis em vértebras & $\begin{array}{l}\text { Rio Miranda } \\
\text { Pantanal/MS }\end{array}$ & RESENDE et al. (1996) \\
\hline & Ls & 145 & 183,2 & 0,085 & 3,4553 & 221 & \multirow{2}{*}{$\begin{array}{l}\text { Anéis em espinhos } \\
\text { Distribuição de } \\
\text { freqüência }\end{array}$} & $\begin{array}{l}\text { Rio Cuiabá } \\
\text { Pantanal/MT }\end{array}$ & $\begin{array}{l}\text { Mateus \& Petrere } \\
(2004)\end{array}$ \\
\hline & Lf & 140 & 149,7 & 0,127 & 3,4542 & 1445 & & $\begin{array}{l}\text { Rio Cuiabá } \\
\text { Pantanal/MT }\end{array}$ & Este estudo \\
\hline
\end{tabular}

produção (Wootton 1990). Também é um importante traço da história de vida e, portanto, uma informação que precisa ser conhecida para o sucesso do manejo da pesca, pois é fundamental para o estabel ecimento de medidas que evitem a exploração de indivíduos jovens e a conseqüente redução do estoque desovante (sobrepesca de crescimento). Geralmente este parâmetro é estimado plotando-se o percentual de indivíduos maturos com o comprimento. Porém estes dados nem sempre estão disponíveis para a maioria das espécies. Por outro lado, estudos comparativos têm demonstrado que o tamanho na maturidade é, em geral, positivamente correlacionado com o tamanho assintótico $(\mathrm{L} \infty)$ dentro de uma mesma família ou gênero (Beverton 1992, Stamps et al. 1998). Assim, para facilitar a estimativa de Lm na ausência de dados adequados, Froese \& Binohlan (2000) propuseram uma equação empírica a partir da relação entre $L m$ e o $L \infty$. O modelo proposto explica $88 \%$ da variação interespecifica no $L m$ a partir da variação em $L \infty$.

A aplicação da equação empírica de Froese e Binohlan (2000) para as espécies aqui estudadas indica que Barbado atinge a maturidade relativamente mais cedo, por volta dos dois anos. O Cachara, o Pintado e o Jaú alcançam a maturidade por volta dos quatro anos e meio. Assim, comparativamente, os três últimos bagres podem ser considerados de maturidade tardia, o que é esperado quando se toma em conta suas estimativas dos parâmetros de crescimento, ou seja, alto L $\infty$ e baixo k.
Os Lm estimados aqui estão abaixo do tamanho médio nas capturas para todas as quatro espécies. Para o Babado o tamanho médio nas capturas excede o Lm em 14\%, para o Cachara em $13 \%$, para o Jaú em $25,5 \%$ e para o Pintado em $22,5 \%$. Assim, os dados aqui apresentados indicam que, aparentemente, as quatro espécies não se encontram ameaçadas por sobrepesca de crescimento na bacia do rio Cuiabá.

Além das diferenças entre as espécies na idade e tamanho médio de primeira maturação, diferenças dentro da espécie não são raras. Segundo Wootron (1990) parte desta variação reflete diferenças genéticas, a outra parte é reflexo dos efeitos ambientais. Para o Barbado o Lm varia entre 47,8 cm (Lf) (este estudo) e 57,4 cm (Ls) no rio Paraguai, Corumbá (PeIXer et al. 2006). Para o Cachara $39,25 \mathrm{~cm}$ (Ls) no rio Apure, Venezuela (Reid 1983) e 86,15 cm (Lf) no Rio Amazonas, Peru (García et al. 2001). Para o Jaú entre $87,5 \mathrm{~cm}$ (Lt) no Rio Paraguai (Sı LVA 1986) e $85 \mathrm{~cm}$ (Lf) (este estudo). Para o Pintado entre $61,5 \mathrm{~cm}$ (Ls) no rio Miranda (Resende et al. 1995) e 83,5 cm (Lt) Rio Paraguai (SıLva 1986). Entretanto, é preciso cautela na comparação destas estimativas, pois os diversos autores utilizaram medidas distintas de comprimento (Lf ou Lt ou Ls) (Tab. VI).

WINEMILLER (1989) sugere que os peixes tropicais de ambientes sazonais podem ser agrupados em três grandes grupos: (I) estrategistas de equilíbrio - que estariam associados com populações locais sedentárias com poucas flutuações, prolon- 
gada estação reprodutiva e cuidados com a prole (correspondendo aos K-estrategistas); (II) oportunistas - caracterizados por peixes pequenos, rápida maturação, múltiplas desovas e alta capacidade de recolonização de habitas pertubados (correspondendo aos r-estrategistas); (III) estrategistas sazonais - estes podem atingir grandes tamanhos, têm alta fecundidade e não apresentam cuidados parentais. Estas espécies exploram a expansão periódica do ambiente aquático por apresentarem migração reprodutiva e desova total no inicio do período chuvoso. Portanto, considerando os parâmetros aqui estimados e a característica reprodutiva evidentemente sazonal do Barbado, Cachara, Jaú e Pintado, pode-se incluí-los na categoria de estrategistas sazonais, assim como a grande maioria das espécies de importância comercial da região pantaneira.

Para uma pesca ter uma taxa de exploração sustentável a mai oria dos indivíduos capturados deveria estar dentro de uma amplitude de comprimento próxima ao ótimo. O comprimento ótimo é aquele onde o produto do número de sobreviventes multiplicado pelo seu peso médio resulta na biomassa mais alta (Beverton 1992, Froese \& Binohlan 2000). A pesca do Barbado e do Cachara capturou, em grande parte, indivíduos maiores que o comprimento ótimo, possivel mente porque este coincide com o tamanho mínimo de captura permitido. Já para o Jaú e o Pintado o tamanho médio dos indivíduos capturados é muito próximo ao tamanho ótimo. Isso implica em que as pescarias do Barbado e do Cachara são menos produtivas que aquelas do
Jaú e do Pintado. Além disso, a pressão de pesca exercida sobre o estoque do Barbado e do Cachara está incidindo mais fortemente sobre os indivíduos maiores que, teoricamente, seriam os mais fecundos (Roff 1988, W INEMILLER \& Rose 1992). Pesquisas recentes vêm apontando que um esforço de pesca exagerado sobre os maiores indivíduos do estoque é uma das principais causas de redução no tamanho dos estoques de peixes marinhos e de água doce e conseqüente redução na produtividade das pescarias (LONGHURST 2002).

\section{Recrutamento}

O processo de recrutamento representa uma interação dinâmica entre a regulação populacional e a variabilidade ambiental. Como foi mostrado, o Cachara e o Jaú apresentam um único pulso de recrutamento enquanto que o Barbado e o Pintado apresentam dois pulsos anuais. Apesar das limitações impostas pelo método de análise do padrão de recrutamento, alguma inferência pode ser feita a partir deste resultado. Desse modo, considerando que para as quatro espécies o evento reprodutivo é anual e restrito a um determinado período, ou seja, os indivíduos de uma coorte têm seu nascimento aproximadamente no mesmo período de tempo, mais de um pulso no recrutamento poderia representar maior variabilidade individual no crescimento, ou então a diferença, em termos de velocidade de crescimento, entre os indivíduos nascidos no inicio do período reprodutivo e aqueles que nasceram ao seu final.

Tabela VI. Quadro comparativo do comprimento de primeira maturação $(\mathrm{Lm}, \mathrm{cm})$ relatado por outros trabalhos com aquele estimado neste estudo para os quatro grandes bagres da bacia do rio Cuiabá, Mato Grosso.

\begin{tabular}{|c|c|c|c|c|c|}
\hline Espécie & L & \multicolumn{2}{|c|}{$\operatorname{Lm}(\mathrm{cm})$} & Local & Fonte \\
\hline \multirow[t]{2}{*}{ P. pirinampu } & Ls & $\begin{array}{l}q^{9} \\
0^{x}\end{array}$ & $\begin{array}{l}43,0 \\
45,6\end{array}$ & Rio Paraguai - Pantanal/MS & Pexer et al. (2006) \\
\hline & Lf & & 47,8 & Rio Cuiabá - Pantanal/MT & Este estudo \\
\hline \multirow[t]{7}{*}{ P. fasciatum } & Ls & $\begin{array}{l}q^{2} \\
\sigma^{x}\end{array}$ & $\begin{array}{l}39,5 \\
39,0\end{array}$ & Rio Apure - Venezuela & Red (1983) \\
\hline & Lf & $\begin{array}{l}q \\
\sigma^{\pi}\end{array}$ & $\begin{array}{l}89,8 \\
82,5\end{array}$ & Rio Amazonas (porção peruana) & García et al. (2001) \\
\hline & Ls & $\begin{array}{l}q \\
\sigma^{\pi}\end{array}$ & $\begin{array}{l}55,0 \\
73,0\end{array}$ & Rio Mamoré - Amazônia Boliviana & Loubens \& PanfiL (2000) \\
\hline & Ls & $\begin{array}{l}q \\
\sigma^{x}\end{array}$ & $\begin{array}{l}63,0 \\
57,0\end{array}$ & Rio Miranda - Pantanal/MS & RESENDE et al. (1995) \\
\hline & Ls & $\begin{array}{l}\text { क } \\
0^{\pi}\end{array}$ & $\begin{array}{l}82,0 \\
60,0\end{array}$ & Alto rio Meta & RAmíREZ \& AlıACO (1995) \\
\hline & Ls & $\begin{array}{l}q \\
\sigma^{x}\end{array}$ & $\begin{array}{l}84,5 \\
61,5\end{array}$ & Rio Mamoré - Amazônia Boliviana & VALDERRAMA et al. (1988) \\
\hline & Lf & & 62,6 & Rio Cuiabá - Pantanal/MT & Este estudo \\
\hline \multirow[t]{2}{*}{ P. luetkeni } & Lt & & 87,5 & Rio Paraguai - Pantanal/MS & SILVA (1986) \\
\hline & Lf & & 85,0 & Rio Cuiabá - Pantanal/MT & Este estudo \\
\hline \multirow[t]{3}{*}{ P. corruscans } & Ls & $\begin{array}{l}\text { क } \\
\sigma^{\pi}\end{array}$ & $\begin{array}{l}66,0 \\
61,5\end{array}$ & Rio Miranda - Pantanal/MS & RESENDE et al. (1995) \\
\hline & Lt & & 83,5 & Rio Paraguai - Pantanal/MS & SILVA (1986) \\
\hline & Lf & & 75,0 & Rio Cuiabá - Pantanal/MT & Este estudo \\
\hline
\end{tabular}




\section{Mortalidade}

Dos parâmetros vitais de populações de peixes exploradas, os componentes da mortalidade são os mais difíceis de se estimar (PALOHEımo 1980). Certamente, M é um dos parâmetros para o qual é mais difícil de se obter uma boa estimativa e freqüentemente é a maior fonte de incerteza na avaliação de estoques (PAuly 1980, Vetter 1987). Por outro lado este é um valor necessário para a maioria dos modelos de dinâmica de estoques. As muitas limitações práticas associadas às estimativas de $M$ têm levado os biólogos pesqueiros a buscar modelos empíricos, a partir de estimativas de parâmetros relacionados a M (Beverton \& Holt 1959, Beverton 1963, Gunderson 1980, Pauly 1980). O modelo empírico de PAULY (1980) parece ser razoável para se estimar $M$, uma vez que é simples, requer poucos parâmetros e foi formulado com base em 175 estoques de diferentes taxas e ambientes, inclusive algumas espécies de água doce (Jones 1984, Gayanilo \& Pauly 1997).

Finalmente é preciso discutir a suposição de que $\mathrm{M}$ permanece constante em todos os grupos de idade (ou comprimento), o que é assumido na maioria dos modelos de dinâmica. Parece ser acordo geral que $M$ é, provavelmente, alta durante estágios juvenis iniciais, baixa no inicio da vida adulta e aumenta com a senescência (Hampton 2000, Conover \& Munch 2002). Segundo Vetter (1988), assumir M constante pode ter sérias conseqüências para o manejo da pesca. No entanto o mesmo autor considera que se $\mathrm{M}$ é relativamente pequeno (não mais que 0,5/ano) e pouco variável, o efeito da imprecisão da estimativa pode ser de pouca importância. Assim, parece que a estimativa de $M$ em grupos onde ela é baixa e pouco variável, é mais provável de ser acurada que estimativas de $M$ em grupos onde ela é alta e variável. Além disto, a suposição $M$ independente da idade parece ser particularmente verdadeira para análises de estoques comerciais, que requerem estimativas de $M$ apenas para idades pós-recrutamento (VETTER 1988).

No caso dos bagres aqui estudados, apenas o Barbado apresentou uma mortalidade natural um pouco acima de 0,5/ano $(0,58)$. Porém, não há informações para se discutir a sua variabilidade. No entanto, considerando que a pesca está interessada em indivíduos pós-recrutamento pesqueiro que, neste caso, são animais de tamanho relativamente grande e predadores, supõese que a mortalidade não varie muito com o tamanho e que a suposição de $M$ constante, não seja tão inconsistente. Mesmo assim, não há dúvidas de que informações acerca da variabilidade de $\mathrm{M}$ com o tamanho sejam fundamentais para a avaliação e manejo dos estoques dessas e outras espécies.

Os métodos utilizados para a estimativa de $Z$ pertencem ao grupo baseado no comprimento médio de uma amostra de peixes capturados e em estimativas dos parâmetros $k$ e $L \infty$ da curva de crescimento de von Bertalanffy. Estes métodos assumem que a partir de um certo tamanho (LC) todos os peixes têm a mesma probabilidade de captura (recrutamento knife-edge) (Gallucci et al. 1996, Sparre \& Venema 1997). Algumas vezes Lc é referido como o comprimento do menor peixe capturado, mas isto provavelmente é um equivoco, como discutido por JONES (1984), pois pode levar a adoção de comprimentos não completamente recrutados. Assim optou-se por usar o Lc médio que representaria o comprimento médio dos peixes recrutados.

A decisão sobre qual destes métodos é o mais adequado para a estimativa de $\mathrm{F}$ para as espécies aqui estudadas não é uma tarefa fácil, pois os métodos baseados em comprimento médio apresentam a mesma limitação básica: a suposição de um sistema de parâmetros constantes. Desse modo não há um critério objetivo de escolha. Poderia se adotar uma posição mais conservadora, optando-se pelo método que estimou o maior valor de Z, por exemplo. Ou então uma posição mais “otimista" e adotar o método que estimou o menor Z. Como qualquer uma dessas posições seria arbitrária neste trabal ho a opção foi usar a média de $Z$ (pois o valor mais provável de uma grandeza é a sua média) a partir dos valores estimados pelos métodos baseados no comprimento médio. Além disso, na biologia pesqueira o que importa não são os valores absolutos, mas sim as tendências gerais.

A taxa de sobrevivência do Barbado é a mais baixa, mesmo não se considerando a mortalidade por pesca. Isso implica em dizer que para um dado número de indivíduos recrutados, a chance de que estes atinjam comprimentos máximos, que corresponderiam a indivíduos mais velhos, são menores que para as outras espécies em questão. Quando se consi dera a mortalidade por pesca a chance de sobrevivência diminui ainda mais e em maior porcentagem para o Barbado, potencializando assim a fração de indivíduos que morrem de um intervalo de tempo para outro.

Obviamente, quanto maior o número de indivíduos recrutados, maior será o número de indivíduos que atingem tamanhos maiores (mais velhos) no estoque. Portanto, para se assegurar isto se deve garantir um recrutamento tão grande quanto possível. Porém, a variabilidade inter-anual do recrutamento não está sob o controle humano, pois este evento é resultado de várias interações bióticas e abióticas muito complexas. Assim, a solução mais plausível para aumentar o recrutamento seria diminuir o F, por meio da diminuição do esforço pesqueiro sobre o estoque.

Para as outras espécies a pesca também reduz a expectativa de permanência da coorte no estoque, porém em uma porcentagem relativamente menor. Desse modo, o que se pode inferir é que a mortalidade por pesca atual afeta o estoque do Cachara, do Jaú e do Pintado de forma mais branda, permitindo que suas coortes permaneçam no estoque por um tempo maior, considerando o mesmo número de recrutas.

Por outro lado é preciso ter em mente que a suposição assumida até aqui é de $M$ constante, no mínimo a partir do recrutamento. Assim o F potencializaria a mortalidade total de forma aditiva. Porém, considerando que $\mathrm{M}$ pode aumentar com a idade, o fato de indivíduos mais velhos serem mortos pela pesca pode não ter um efeito negativo na população uma vez que se esta retirando indivíduos que morreriam por outras cau-

Revista Brasileira de Zoologia 24 (1): 87-98, março 2007 
sas associadas a senescência, com uma taxa maior, o que seria compensado por $\mathrm{F}$.

No entanto, se a predação é a principal fonte de $M$, para estas espécies esta não é esperada aumentar tanto com a idade (comprimento), pois todas no recrutamento são relativamente grandes, tendo uma chance reduzida de serem predadas. Assim a análise do quanto $\mathrm{F}$ afetaria o estoque tem que ser feita em conjunto com outros fatores indicativos da saúde do estoque.

\section{AGRADECIMENTOS}

Aos pescadores do rio Cuiabá e aos comerciantes de peixes do mercado "Antônio Moysés Nadaf" pelo apoio durantea obtenção dos dados. À Fundação de Amparo à Pesquisa do Estado de Mato Grosso, ao Centro de Pesquisas do Pantanal e ao $\mathrm{CNPq}$, pelo continuado apoio financeiro às pesquisas do Laboratório de Ecologia e Manejo de Recursos Pesqueiros.

\section{REFERÊNCIAS}

Albuquerque, S. P.; F.L.R. Campos \& A.C. Catella. 2003. Sistema de Controle da Pesca de Mato Grosso do Sul SCPESCA/ MS 9 - 2002. Corumbá, EMBRAPA, CPAP Boletim de Pesquisa $47,57 p$.

Ault, J.S. \& N.M. Ehrhardt. 1991. Correction to Beverton and Holt Z-estimator for truncated catch length-frequency distribution. ICLARM Fishbyte 9: 37-39.

Bayley, P.B. \& M. Petrere JR. 1989. Amazon fisheries: assessment methods, current status and management options, p. 385398. In: D.P. Dodge (Ed.). Proceedings of the International Large River Symposium (LARS). Canadian Special Publication of Fisheries and Aquatic Sciences, vol. 106, 628p.

BertalanfFy, L. von. 1938. A quantitative theory of organic growth. Human Biology 10: 181-213.

BeVERTON, R.J.H. 1963. Maturation, growth and mortality of clupeid and engraulid stocks in relation to fishing. Rapports et Procés-Verbaux Des Réunions, Conseil International Pour l'Exploration de la Mer 154: 44-67.

Beverton, R.J.H. 1992. Patterns of reproductive strategy parameters in some marine teleost fishes. Journal of Fish Biology 41: 137-160.

Beverton, R.J.H. \& S.J. Holt. 1959. A review of the lifespans and mortality rates of fish in nature, and their relation to growth and other physiological characteristics. Ciba Foundation Colloquium on Ageing 68: 142-180.

Conover, D.O. \& S.B. Munch. 2002. Sustaining fisheries yields over evolutionary time scales. Science 297: 94-96.

DeAngelis, D.L.; K.A. Rose; L.B. Crowder; E.A. Marschall \& D. LIKA. 1993. Fish cohort dynamics: application of complementary modeling approaches. American Naturalist 142: $604-$ 622.

Froese, R. \& C. Binohlan. 2000. Empirical relationships to estimate asymptotic length, length at first maturity and length at maximum yield per recruit in fishes, with a simple method to evaluate length frequency data. Journal of Fish Biology 56, 758-773.

Gallucci, V. F; B. Amjoun; J. Hedgepeth \& H.L. Lal. 1996. Sizebased methods of stock assessment of small-scale fisheries, p. 9-81. In: V.F. GallucCl; B.S. SAila; D.J. Gustafson \& B.J. RotHSCHILD. (Eds). Stock assessment: quantitative methods and applications for small-scale fisheries. New York, Lewis Publishers, 544p.

García, A.; V. Montreuil \& R. Rodríguez. 2001. Talla dela primera maturación y época de desova de la "doncella", Pseudoplatystoma fasciatum (Linnaeus), y el "tigre zúngaro", Pseudoplatystoma tigrinum (Valenciennes), en la Amazonía Peruana. Boletim do Museu Paraense Emílio Goeldi, Série Zoologia 17: 3-13.

Gayanilo JR, F.C. \& D. Pauly. 1997. The FAO-ICLARM Stock Assessment Tools (FISAT) Reference manual. Roma, FAO Computerized Information Series (Fisheries) 8, 262p.

Gayanilo JR, F.C.; P. Sparre \& D. Pauly. 1994. The FAO-ICLARM Stock Assessment Tools (FISAT) User's Guide. Roma, FAO Computerized Information Series (Fisheries) 7, 124p.

GundeRSON, D.R. 1980. Using r-K selection theory to predict natural mortality. Canadian Journal of Fisheries and Aquatic Science 37: 2266-2271.

HAMPTON, J. 2000. Natural mortality rates in tropical tunas: size really does matter. Canadian Journal of Fisheries and Aquatic Science 57: 1002-1010. 2000.

HILBORN, R. \& C.J. W ALTERS. 1992. Quantitative fisheries stock assessment: choice, dynamics and uncertainty. New York, Chapman and Hall, 570p.

ISAAC, V.J. 1990. The accuracy of some length-based methods for fish population studies. ICLARM Technical Report 27: $1-81$.

JobLING, M. 1995. Environmental biology of fishes. London, Chapman \& Hall, 455p.

JONES, R. 1984. Assessing the effects of changes in exploitation pattern using length composition data. FAO Fisheries Technical Paper 256: 118p.

KING, M. 1995. Fisheries biology, assessment and management. Oxford, Fishing News Book Blackwell Science, 341p.

LONGHURST, A. 2002. Murphy' law revisited: Iongevity as a factor in recruitment to fish populations. Fisheries Research 56: 125-131.

Loubens, G. \& J. Panfili, J. 2000. Biologie de Pseudoplatystoma fasciatum et Pseudoplatystoma tigrinum (Teleostei: Pimelodidae) dans lê bassin du Mamoré (Amazonie Bolivienne). Ichthylogical Exploration of Freshwaters 11: 13-34.

Lundberg, J.G. \& M.W. Littmann. 2003. Pimelodidae (Longwhiskered catfishes), p. 432-446. In: R.E. ReIS; S.O. KulLander $\&$ C.J. FerRARIS JR (Eds). Checklist of the freshwater fishes of South and Central América. Porto Alegre, Edipucrs, $729 p$.

Mateus, L.A.F.; J.M.F. Penha \& M. Petrere JR. 2004. Fishing resources in the rio Cuiabá basin, Pantanal do Mato Grosso, 
Brazil. Neotropical Ichthyology 2 (4): 217-227.

Mateus, L.A.F. \& M. Petrere JR. 2004. Age, growth and yield per recruit analysis of the pintado, Pseudoplatystoma corruscans (Agassiz, 1829) in the Cuiabá River basin, Pantanal Matogrossense, Brazil. Brazilian Journal of Biology 64 (2): 257264.

NovoA, D.R. 1982. Los Recursos pesqueros Del río Orinoco y su explotación. Caracas, Corporación Venezolana de Guayana, 386p.

Paloheimo, J.E. 1980. Estimation of mortality rates in fish populations. Transactions of American Fishery Society 109: 378-386.

PAULY, D. 1979. Theory and management of tropical multispecies stocks. Manila, ICLARM Studies and Reviews 1, 35p.

PAULY, D. 1980. On the interrelationships between natural mortality, growth parameters and mean environmental temperature in 175 fish stock. Journal du conseil international pour l'exploitation de la mer 39 (2): 175-192.

PAULY, D. \& J.L. MunRo. 1984. Once more on the comparison of growth in fish and invertebrates. ICLARM Fishbyte 2 (1): 1-21.

Peixer, J.; L.A.F. Mateus \& E. Resende. 2006. First gonadal maturation of Pinirampus pirinampu (Siluriformes: Pimelodidade) in the Pantanal, Mato Grosso do Sul state, Brazil. Brazilian Journal of Biology 66 (1B): 317-323.

Penha, J.M.F. \& L.A.F. Mateus. 2007. Sustainable harvest of two large predatory Catfish in the Cuiabá river basin, northern Pantanal, Brazil. Brazilian Journal of Biology 67 (1): 631637.

Petrere, M. Jr; A.A. Agostinho; E.K. Okada \& H.F. Julio Jr. 2002. Review of thefisheries in the Brazilian portion of the Paranál Pantanal basin, p. 123-143. In: I.G. Cowx (Ed). Management and ecology of lake and reservoir fisheries. Londres, Fishing News Books, 416p.

Quinn II, T.J. \& R.B. Deriso. 1999. Quantitative fish dynamics. New York, Oxford University Press, 542p.

REID, S. 1983. La biología de los bagres rayados Pseudoplatystoma fasciatum y P. tigrinum en la cuenca del río Apure, Venezuela. Venezuela. Revista Unellez de ciencia y Tecnología 1: 1341.

Reina, M.P.; H. Ramírez \& M. Barco. 1995. Edad y crecimiento de Paulicea lüetkeni (Steindachner, 1876) (Pisces: Pimelodidae) basado en la lectura de estructuras duras (espinas de aleta pectoral) en el alto río Meta (Colombia). Boletín Científico INPA 3: p.115-130.

Resende, E.K.; A.C. Catella; F.L. Nascimento; S.S. Palmeira; R.A. Candido; M.S. Lima \& V.L.L Almeida. 1996. Biologia do curimbatá (Prochilodus lineatus), pintado (Pseudoplatystoma corruscans) e cachara (Pseudoplatystoma fasciatum) na bacia hidrográfica do rio Miranda, Pantanal, Mato Grosso do Sul, Brasil. Corumbá, EMBRAPA, CPAP Boletim de Pesquisa 2, 75p.

Roff, D.A. 1988. The evolution of migration and some life history parameters in marine fish. Environmental Biology of Fishes 22: 133-146.

Rosenberg, A.A. \& J.R. Beddington. 1987. Monte-Carlo testing of two methods for estimating growth from length-frequency data with general conditions for their applicability, p. 283298. In: D. Pauly \&. G.R. Morgan (Eds). Length based methods in fisheries research. Manila, ICLARM Conference Proceedings 13, 468p.

Ruffino, M. \& V.J. ISAAC. 1995. Life cycle and biological parameters of several Brazilian Amazon fish species. NAGA The ICLARM Quarterly 18: 41-45.

SHEPHERD, J. G. 1987. A weakly parametric method for estimating growth parameters from length composition data, p. 113119. In: D. PAULY \&. G.R. MORGAN (Eds). Length based methods in fisheries research. Manila, ICLARM Conference Proceedings 13, 468p.

SILVA, M.V. 1986. Mitos e verdades sobre a pesca no Pantanal sul mato-grossense. Campo Grande, FIPLAN, 146p.

SPARRRE, P. \& S.C. VENEMA. 1997. Introdução à avaliação de mananciais de peixes tropicais. Parte 1: Manual. Roma, FAO, 404p.

Ssetongo, G.W. \& P.A.LARkin. 1973. Some simple methods of estimating mortality rates of exploited fish populations. Journal of Fishery Research Board of Canada 30: 695-698.

Stamps, J.A.; M. Mangel \& J.A. Phillips. 1998. A new look at relationships between size at maturity and asymptotic size. American Naturalist 152: 470-479.

Valderrama, M.; M. Zarate; G. Vera; C. Moreno; P. Caraballo \& J. MARTINEZ. 1988. Determinación de la talla media de madurez y análisis de la problemática con referencia a las tallas medias de captura del bagre rayado (Pseudoplatystoma fasciatum) Linnaeus 766 (Pisces: Pimelodidae) en la cuenca del río Magdalena, Colombia. Trianea Acta Cientifica y Tecnologica Inderena 2: 537-549.

VETTER, E. F. 1988. Estimation of natural mortality in fish stocks: a review. Fishery Bulletin 86: 25-43.

Wetherall, J.A.; J.J. Polvina \& S. Ralston. 1987. Estimating growth and mortality in stead-state fish stock from length-frequency data, p. 53-74. In: D. Pauly \& G.R. Morgan (Eds). Length based methods in fisheries research. Manila, ICLARM Conference Proceedings 13, 468p.

Winemiller, K.O. 1989. Patterns of variation in life history among South American fishes in seasonal environments. Oecologia 81: 225-241.

Winemiller, K.O. \& K.A. Rose. 1992. Patterns of life-history diversification in North American fishes: implications for population regulation. Canadian Journal of Fisheries and Aquatic Science 49: 2196-2218.

Wootton, R.J. 1990. Ecology of teleost fishes. London, Chapman \& Hall, 403p.

Recebido em 12.V.2006; aceito em 23.II.2007. 